\title{
La bio-ingénierie de la régénération osseuse
}

$>$ Les pertes de substance, surtout lorsqu'elles sont Hervé Petite de grande taille, mettent souvent à mal les capacités naturelles de réparation de l'os. Leur traitement repose alors essentiellement sur l'apport d'os autologue. Cependant, l'autogreffe osseuse n'est pas toujours possible. Le prélèvement ne peut en effet se faire qu'en quantité limitée et engendre une morbidité non négligeable. Le chirurgien peut utiliser alternativement des alloimplants ou des biomatériaux ostéoconducteurs. Ces matériaux ne rendent hélas des services que pour le comblement de défauts de petite taille. Biologiquement «muets », ils ne servent que de support passif à la formation osseuse. Ces limites ont incité les chercheurs à développer des substituts du tissu osseux ayant un fort pouvoir ostéogène conféré par l'intermédiaire de facteurs de croissance ou de cellules ostéocompétentes. Des études cliniques prospectives doivent être effectuées afin d'évaluer la place de ces nouvelles techniques dans l'arsenal thérapeutique du chirurgien orthopédiste. <

L'une des propriétés les plus remarquables des êtres vivants est la capacité de certains tissus de s'auto-réparer. L'exemple le plus impressionnant chez les vertébrés est représenté par la régénération d'un membre chez les urodèles [1]. Chez les mammifères, cette propriété est l'apanage d'aires déterminées telles que l'extrémité des doigts chez le souriceau [2]. Le plus souvent, toutefois, Ia lésion d'un organe entraîne généralement une cascade biologique conduisant à la fermeture de la plaie par un tissu fibreux. Au cours de l'évolution, a-t-il été plus avantageux de perdre cette capacité de régénération au profit d'une fermeture rapide de la plaie? La question reste entière. Dans une certaine mesure, l'os échappe à cette règle puisqu'il est capable de s'auto-réparer à condition que la lésion soit de petite taille. La technique de « distraction » osseuse, qui permet l'allongement de membre, illustre de façon spectaculaire ce potentiel d'auto-répa-

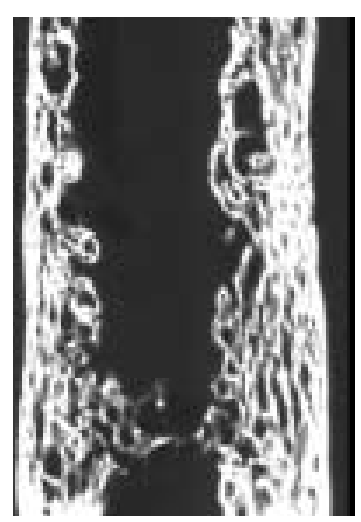

ration (Figure 1). À la suite d'une fracture, on observe une cascade d'événements reproduisant, chez le sujet adulte, l'ostéogenèse de l'embryon. Le processus biologique de réparation osseuse est généralement suffisamment efficace pour que l'intervention humaine se limite à un réalignement et à une immobilisation du membre fracturé, suivis ou non de rééducation fonctionnelle. D'ailleurs, sur les 6 millions de fractures observées chaque année aux États-Unis, des complications ne surviennent que dans 5 à $10 \%$ des cas, entraînant un retard, voire une absence de consolidation, à la suite de défaillances de nature mécanique ou biologique [3]. L'absence de consolidation est souvent due à la formation d'un mélange de tissus fibreux et osseux ne permettant pas la restauration de la continuité osseuse.

De surcroît, les avancées thérapeutiques en orthopédie mettent souvent à mal les capacités naturelles de réparation de l'os. En effet, le traitement de tumeurs, de kystes ou de foyers infectieux nécessite souvent l'exérèse de pièces osseuses de taille trop importante pour que le processus naturel d'auto-réparation puisse, à lui seul, induire une consolidation osseuse. Le comblement de ces défauts est alors généralement assuré par une autogreffe. La 
quantité de greffon autologue étant cependant limitée, le chirurgien peut alors greffer soit du tissu allogénique, soit des matériaux de substitution osseuse. Ces matériaux sont biologiquement muets et ne servent, au mieux, que de support passif à la réparation osseuse. On dit qu'ils sont « ostéoconducteurs ». L'enrichissement de ces matériaux avec des protéines intervenant dans la morphogenèse osseuse (BMP, bone morphogenic proteins) $(\rightarrow$ ) permet de $\rightarrow) \mathrm{m} / \mathrm{s} \quad$ rendre ces matériaux biologiquement actifs. On dit qu'ils 1996, n³, sont «ostéo-inducteurs 》 car les BMP, libérées localement p. 364

$(\rightarrow) \mathrm{m} / \mathrm{s}$ 2001, n ${ }^{\circ} 12$, p. 1252

$\rightarrow) \mathrm{m} / \mathrm{s}$ 2001, $\mathrm{n}^{\circ} 12$, p. 1260

par le biomatériau, vont induire le recrutement local de cellules mésenchymateuses et permettre le déclenchement de la cascade biologique conduisant à la formation osseuse. Enfin, la possibilité de combiner ces matériaux avec des cellules ostéocompétentes, obtenues à partir d'un simple prélèvement de moelle osseuse, a permis de rendre ces matériaux directement ostéogènes. Nous relaterons ici successivement les diverses propriétés de ces matériaux en débutant par l'autogreffe qui est la technique de référence (Tableau I), la conception des autres matériaux découlant de la compréhension de son fonctionnement.

\section{Autogreffe osseuse}

Le principe consiste en la transplantation d'une pièce osseuse là où le comblement est nécessaire. La pièce osseuse est prélevée soit en zone spongieuse, soit en zone corticale ou encore cortico-spongieuse si l'on attend du greffon qu'il possède également des propriétés mécaniques intéressantes [4]. L'os est généralement prélevé dépourvu de sa vascularisation. Les sites donneurs de prédilection sont les crêtes iliaques antérieures et postérieures. La réaction que va entraîner la mise en place du greffon est un véritable partenariat entre le transplant et les tissus environnants receveurs. Elle dépend de trois propriétés : l'ostéoconductivité, l'ostéoinduction et le potentiel ostéogène, propriété particulièrement importante lorsque le greffon autologue a conservé sa vascularisation.

L'autogreffe n'entraîne évidemment aucune réaction immunologique. Cependant, parmi les cellules greffées, peu survivent à la transplantation. Les greffons spongieux, revascularisés plus rapidement que les greffons corticaux, permettent la survie d'une fraction plus importante de cellules. L'incorporation de la greffe s'effectue en trois étapes [5]. Lors de la transplantation d'un greffon spongieux non vascularisé chez le chien, on assiste dans les trois premières semaines à son invasion par une néoangiogenèse et à sa colonisation par des cellules du mésenchyme provenant des tissus receveurs environnants. Entre 3 et 12 semaines, les trabécules osseux du greffon servent de support aux cellules mésenchymateuses qui se sont différenciées en ostéoblastes $(\rightarrow)$ et synthétisent la matrice osseuse. Simultanément, des ostéoclastes $(\rightarrow)$ résorbent les trabécules osseux nécrotiques du greffon. Entre 3 et 6 mois, le greffon est remodelé, entraînant une réorientation des trabécules en fonction de l'environnement mécanique. Dans ce modèle, le remodelage cesse après un an.

Malgré son intérêt, l'autogreffe possède un certain nombre d'inconvénients pour le patient, le principal étant la nécessité d'un second site opératoire, ce qui peut entrâ̂ner une morbidité incluant des douleurs importantes, des hématomes, des abcès et des névralgies. Elle n'est en outre pas toujours possible puisque la taille du greffon prélevable est limitée.

\section{Implant osseux allogénique}

L'implant osseux allogénique est obtenu soit à l'occasion d'une arthroplastie totale de hanche, soit au cours de prélèvements multiples [4]. Sa mise en forme et sa conservation sont effectuées par les banques d'os (en France, ce sont des établissements publics ou privés).

Pour l'instant, il n'existe pas d'harmonisation des procédures en matière de nettoyage et de préparation des implants. Le décret du 30 août 1999 fixe les conditions d'obtention des « autorisations d'établissements », c'està-dire les critères auxquels doivent répondre les banques de tissus en matière de personnels, de locaux et de matériels. Ces autorisations émanent de la Direction de l'Inspection des Établissements. Un second décret qui fixera les critères d'obtention des autorisations « procédés-produits », c'est-à-dire les critères auxquels doivent

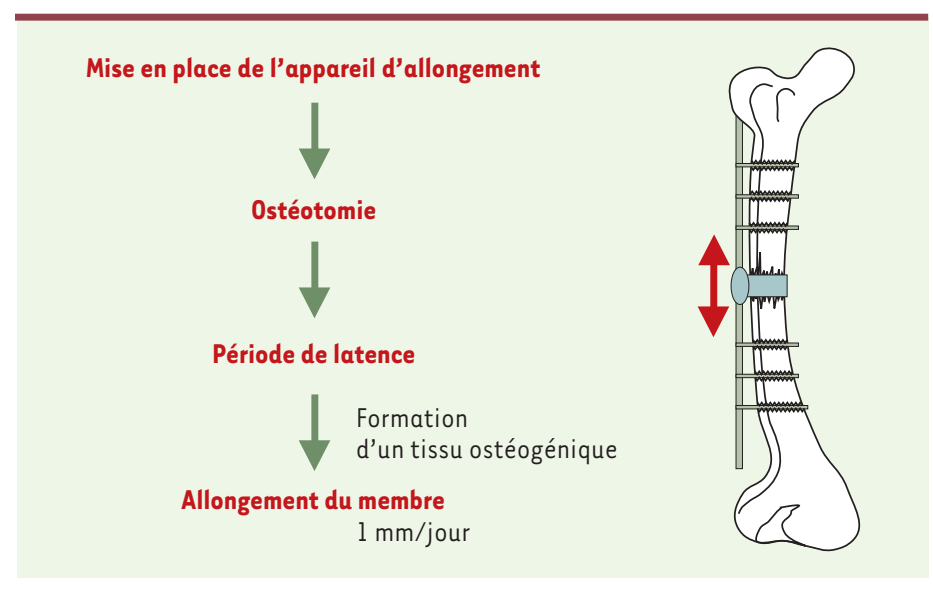

Figure 1. Technique de dis-traction osseuse. Après section de la corticale osseuse et formation d'un tissu ostéogène, on exerce un allongement progressif du membre. La perte de substance ainsi créée est comblée par un tissu osseux néoformé. On peut de cette manière obtenir un allongement de plusieurs centimètres. Le temps de consolidation est chiffré à environ un mois par centimètre gagné. 
répondre les procédés et les produits en matière de qualité, de sécurité et d'efficacité, devrait être publié prochainement. L'évaluation dépendra de la Direction de l'ćvaluation des Médicaments et des Produits Biologiques.

En fonction de l'utilisation finale, les allo-implants soit sont cryoconservés directement à $-80^{\circ} \mathrm{C}$ sans autre traitement, soit ils subissent un procédé d'inactivation virale pouvant inclure un protocole de délipidation à chaud ou à froid. Ce traitement permet l'élimination des cellules osseuses, cartilagineuses, vasculaires et hématopoïétiques présentes dans l'implant allogénique et dont les glycoprotéines membranaires sont particulièrement immunogènes. Une irradiation par rayonnement $\gamma$ peut être effectuée après lyophilisation de la préparation.

La réaction immunologique au greffon allogénique, essentiellement à médiation cellulaire, est influencée par le mode de préparation et de stockage du greffon, le degré de compatibilité HLA entre le greffon et le receveur et l'immunocompétence du receveur. L'effet principal de cette réaction immunologique serait d'inhiber ou de retarder la vascularisation du greffon ou sa colonisation par les cellules osseuses et les ostéoclastes. Malgré ces réserves, les allo-implants sont utilisés couramment, qu'il s'agisse d'un greffon unique ou de fragments multiples, lors de reprises de prothèses articulaires afin de combler des pertes de substance liées à une ostéolyse autour de la prothèse, ou dans le cadre de la reconstruction de grandes pertes de substance osseuse, par exemple après l'exérèse de tumeurs.

\begin{tabular}{lccc}
\hline & $\begin{array}{l}\text { Ostéo- } \\
\text { conduction }\end{array}$ & $\begin{array}{l}\text { Ostéo- } \\
\text { induction }\end{array}$ & $\begin{array}{c}\text { Potentiel } \\
\text { ostéogène }\end{array}$ \\
\hline Autogreffe & + & + & + \\
\hline Implant osseux allogénique & + & - & - \\
\hline Substituts osseux & + & - & - \\
\hline Matériau + BMP & + & + & - \\
\hline Matériau + cellules ostéogènes & + & - & + \\
\hline
\end{tabular}

Tableau I. Propriétés des différents matériaux de comblement. Un matériau ostéoconducteur est un matériau qui sert de support passif à la repousse osseuse. Un matériau ostéogène est un matériau possédant des cellules ostéocompétentes capables de former un tissu osseux. Un matériau ostéoinducteur est un matériau contenant des BMP, molécules qui vont recruter localement des cellules mésenchymateuses indifférenciées et provoquer leur différenciation en cellules ostéocompétentes. II faut remarquer que l'ostéo-induction dépend de la présence des cellules mésenchymateuses indifférenciées capables de répondre à la stimulation des BMP. Le potentiel ostéogène dépendra de la survie des cellules ostéogènes dans l'implant donc de la rapidité de l'envahissement vasculaire.

\section{Substituts osseux}

On distingue deux catégories de biomatériaux selon les relations qui s'établissent entre le biomatériau et le tissu osseux (pour revue, voir [4, 6-8]).

- Les matériaux « biotolérés » (exemple : polyméthylméthacrylate) dont l'incorporation à l'os s'effectue par l'intermédiaire d'une couche fibreuse.

- Les matériaux bioactifs (les céramiques à base de phosphate de calcium, les bioverres, les carbonates de calcium...) établissent un lien direct avec l'os sans tissu fibreux intermédiaire. En pratique, lorsqu'on implante ces matériaux dans un site osseux, l'énergie nécessaire à leur extraction est forte pour les matériaux bioactifs et faible pour les matériaux biotolérés.

\section{Matériaux bioactifs}

\section{Céramiques à base de phosphate de calcium}

Le tissu osseux est un matériau comprenant une phase organique (30 à $40 \%$ du poids $\mathrm{sec}$ ) et une phase minérale sous forme de phosphate de calcium apatitique (60 à $70 \%$ du poids sec). L'ostéoconductivité d'un greffon autologue résiderait dans la composition chimique de sa phase minérale, ce qui a conduit les chercheurs à proposer l'utilisation des phosphates de calcium en orthopédie. L'hydroxyapatite est, sur le plan chimique, le phosphate de calcium qui se rapproche le plus de la phase minérale de l'os. Cependant, alors que les hydroxyapatites stœchiométriques $\left[\mathrm{Ca}_{10}\right.$, $\left.\left(\mathrm{PO}_{4}\right)_{6},(\mathrm{OH})_{2}\right]$, contiennent $39,9 \%$ de $\mathrm{Ca}, 18,5 \%$ de $\mathrm{P}$ et $3,38 \%$ d'OH, les hydroxyapatites naturelles contiennent aussi de petites quantités d'ions carbonate, magnésium, sodium et des éléments tels que fer et chlore à l'état de traces. Ces substitutions peuvent avoir des répercussions sur la solubilité du composé. Par exemple, plus le taux d'ions carbonates est élevé, plus l'hydroxyapatite est soluble (pour revue, voir [8]).

L'hydroxyapatite, le phosphate de calcium tricalcique ( $\beta$ TCP) et des mélanges d'hydroxyapatite- $\beta$ TCP en proportions variables sont les plus couramment utilisés en chirurgie orthopédique $[4,8]$. Ces phosphates de calcium se présentent sous forme de blocs ou de granules. Ils sont fabriqués à partir d'une poudre mise en forme à froid puis chauffée en dessous du point de fusion, entraînant une consolidation de la structure. Ces matériaux peuvent être obtenus sous forme poreuse. La macroporosité (pore > $50 \mu \mathrm{m}$ ) est obtenue en incorporant des billes d'un composé volatile (par exemple de naphtalène) avant le traitement thermique. La microporosité (pore $<10 \mu \mathrm{m}$ ) dépendra de la température et du temps de chauffage de la poudre. Ces matériaux possèdent une bonne résistance à la compression mais leur déformation lors de la rupture est très faible et, par conséquent ils se fracturent facilement lors d'un choc. 


\section{Comportement in vivo}

des céramiques phosphocalciques $[8,10]$

- Activité à l'interface

Lors du contact avec les fluides biologiques, les macrocristaux de la céramique subissent une dissolution partielle, ce qui induit une augmentation locale des concentrations en phosphate de calcium. On assiste alors à une nucléation secondaire dans les espaces entre les cristaux de la céramique et à une croissance hétéroépitaxique à la surface des cristaux résiduels du biomatériau. Les cristaux d'hydroxyapatite carbonatée qui croissent à partir du biomatériau sont aussi associés à la matrice collagénique, créant ainsi une véritable continuité anatomique entre la céramique et le tissu biologique. On parle d'ostéocoalescence [8].

\section{- Activité cellulaire}

Au contact des céramiques à base de phosphate de calcium, on retrouve des cellules à activité résorbante (monocytes, macrophages, cellules géantes et ostéoclastes), des fibroblastes et des ostéoblastes qui synthétisent une matrice ostéoïde de façon polaire en contact direct avec la surface de la céramique. La minéralisation de cette matrice va permettre l'établissement d'un pontage direct entre la céramique et la phase minérale osseuse. Le remodelage de la céramique est effectué par des cellules multinucléées et des ostéoclastes. L'hydroxyapatite n'est dégradable que très lentement in vivo. Les fabricants modulent la vitesse de dégradation des hydroxyapatites en y ajoutant du $\beta$ TCP. Plus le rapport BTCP/ hydroxyapatite est élevé, plus la dissolution est rapide. Un pourcentage de $\beta$ TCP supérieur à $25 \%$ dans les céramiques d'hydroxyapatite assure l'obtention d'une bonne intégration de la céramique dans le tissu osseux. Une porosité > $100 \mu \mathrm{m}$ est nécessaire à une colonisation optimale par le tissu osseux. La vitesse de résorption des céramiques dépend aussi de la densité, de la taille des grains et de la porosité de la céramique.

\section{Matériaux d'origine naturelle et dérivés}

Les implants osseux provenant d'une espèce différente (ou xéno-implants), essentiellement d'origine bovine, subissent un traitement en plusieurs étapes : élimination des débris cellulaires, déprotéinisation plus ou moins complète, délipidation, inactivation virale et prionique et, enfin, stérilisation finale par irradiation [7]. Les xénoimplants peuvent aussi être recouverts de céramique. Le traitement comprend généralement une étape de cuisson, une étape de pyrolyse à $900^{\circ} \mathrm{C}$, puis de céramisation (> 1 $200^{\circ} \mathrm{C}$ ) pendant 3 à 4 jours et de stérilisation aux rayons $\gamma$. L'exosquelette de corail (Biocoral ${ }^{\circledR}$ ) constitué essentiellement de carbonate de calcium et possédant une porosité ouverte est un matériau ostéoconducteur biocompatible. Il est possible, grâce à un processus physico-chimique, de le transformer en hydroxyapatite tout en maintenant l'architecture initiale du corail. On obtient alors un matériau - hydroxyapatite coralliniforme - moins résorbable mais plus fragile mécaniquement [6].

\section{Matériaux ostéo-inducteurs}

\section{Principe}

L'hypothèse sous-jacente à l'utilisation des facteurs de croissance en orthopédie est qu'il est possible d'altérer le cours d'événements clés de la cicatrisation osseuse en modifiant la concentration locale d'un ou de plusieurs facteurs de croissance. Les études du comportement biologique des autogreffes montrent que le développement vasculaire et l'induction de la formation osseuse revêtent une importance fondamentale dans l'intégration des greffons osseux. Les facteurs de croissance favorisant ces phénomènes sont disponibles et ont donc été utilisés expérimentalement pour accélérer ces événements.

Libération de facteurs de croissance à partir d'un support Nous ne relaterons ici que les expériences relatives aux protéines de la morphogenèse osseuse (BMP). Une simple injection de BMP suffit à induire la cascade biologique conduisant à la formation osseuse. Toutefois, deux impératifs - l'action locale de ces molécules et le besoin d'un support physique pour que la formation osseuse puisse avoir lieu - impliquent l'utilisation d'un vecteur de libération (pour revue, voir [11, 12]). Ce vecteur doit éviter une clairance trop rapide du facteur de croissance et permettre sa libération de manière continue et prédictible à des doses thérapeutiques, et cela pendant un temps suffisant. II y a là une contradiction. En effet, la demi-vie de ces molécules est de l'ordre de la minute ou de l'heure, alors que la réparation osseuse prend environ un mois. De plus, le vecteur joue aussi le rôle de support pour la formation osseuse. À ce titre, il doit permettre à la fois la migration, la prolifération et la différenciation des ostéoblastes sur ses surfaces et l'envahissement de sa porosité par le réseau vasculaire.

De très nombreux supports ont été évalués dont des matériaux à base de phosphate de calcium (hydroxyapatite, TCP, ciments phosphocalciques), le corail, des polymères naturels (collagène de type I, fibrine, acide hyaluronique) et synthétiques appartenant essentiellement à la classe des acides poly- $\alpha$-hydroxyl (acides polylactiques et polyglycoliques) ou encore des matériaux composites. Les résultats obtenus avec le collagène de type I sont encourageants $[12,13]$. Pourtant, le vecteur idéal reste à découvrir puisque des milligrammes d'extraits protéiques 
ou des microgrammes de protéines recombinantes sont nécessaires pour induire la réparation osseuse de défauts importants alors que les concentrations de BMP dans l'os sont de l'ordre du microgramme/kg [14]. Enfin, remarquons que l'obtention en clinique d'une formation osseuse robuste nécessitera probablement des doses encore plus élevées si l'on veut tenir compte de l'état physiologique des patients ou encore des différences de potentiel ostéogène selon les sites anatomiques. Ces paramètres peuvent avoir un effet très important sur l'ampleur de la réponse.

De nombreux travaux expérimentaux montrent l'intérêt de ces facteurs de croissance soit sous forme d'extraits, soit sous forme purifiée en chirurgie réparatrice maxillofaciale et en orthopédie. Elles ont été utilisées par exemple pour accélérer la repousse osseuse dans des défauts de taille critique au niveau du crâne chez le rat [15] (Figure 2), de la mandibule chez le chien [16] ou encore des os longs chez le mouton [17].

\section{Thérapie génique ex vivo}

Afin de s'affranchir de ces difficultés, plusieurs groupes ont proposé une approche de thérapie génique pour apporter localement ces facteurs de croissance. Le transgène est introduit dans la cellule hôte soit par une technique ex vivo soit directement in situ (voir plus loin) [12, 18]. La cellule transgénique va agir comme un véritable bioréacteur capable de produire et de délivrer localement des BMP. Dans le cadre de la réparation osseuse, il est sou-
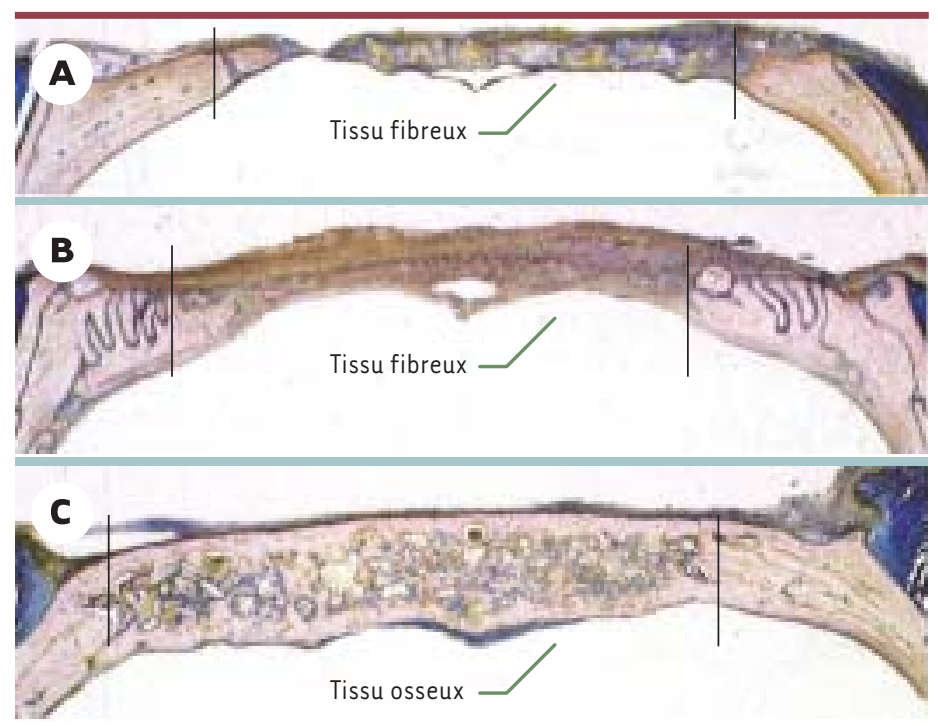

Figure 2. Comblement d'une lésion osseuse au niveau de la calotte crânienne chez le rat. Les défauts laissés vacants (A) ou comblés par du corail (B) sont envahis par un tissu fibreux tandis que les défauts comblés par du corail enrichi par $100 \mu \mathrm{g}$ de BMP extraites d'os bovin sont envahis par un tissu osseux néoformé (C). Sections au niveau du crâne après inclusion en méthacrylate de méthyle et coloration bleu de Stevenel et picro-fuschine de Van Gieson [15]. haitable que le transgène ne soit exprimé que durant quelques semaines.

On prélève chez le patient une population de cellules dont on va amplifier le nombre in vitro et dans lesquelles on insère le gène d'intérêt. La population de ces cellules transgéniques peut être réimplantée après ensemencement sur un support. Le choix de la population cellulaire n'est pas anodin puisque des cellules d'origines différentes vont produire des quantités très différentes du facteur de croissance [19]. Des cellules souches mésenchymateuses (CSM) [20], des fibroblastes dermiques ou des fibroblastes gingivaux [21] ont pu être transfectés avec I'ADNc codant pour la BMP-2 ou la BMP-7. Ces cellules, une fois réimplantées, induisent une formation osseuse. Cette approche est, en pratique, relativement lourde mais présente l'intérêt de fournir trois éléments indispensables à la formation osseuse : des cellules souches, un facteur de croissance capable de stimuler leur différenciation, et un support sur lequel elles vont proliférer et se différencier. Des vecteurs adénoviraux sont de bons candidats pour introduire le transgène dans les cellules puisque l'obtention de titres élevés d'adénovirus recombinant est relativement aisée et que l'efficacité de transfection est élevée même dans les cellules quiescentes. De plus, le transgène, présent sous forme d'épisome, sera perdu au cours des divisions cellulaires. Avec cette approche, on a pu montrer qu'il était possible de réparer des défauts importants au niveau du fémur chez le rat en utilisant des cellules souches mésenchymateuses comme bioréacteur [20]. Cependant, le risque lié à l'utilisation de vecteurs adénoviraux et qui fait perdre, à notre avis, une partie de l'intérêt de cette technique est la réaction immunologique contre les protéines du virus. En outre, les systèmes utilisés jusqu'à très récemment n'offrent pas la possibilité de contrôler les quantités de BMP sécrétées par les cellules, ce qui est problématique.

\section{Transgenèse in situ}

Dans ce cas, le transgène véhiculé par un adénovirus recombinant est injecté directement dans les masses musculaires. Cette technique a été utilisée lors d'arthrodèses vertébrales expérimentales [22, 23]. II y a certes induction de la formation osseuse, mais les études ont été réalisées chez des animaux immunodéprimés. L'impact de la réponse immunitaire sur la formation osseuse lors d'utilisation d'adénovirus est illustrée par l'expérience d'Okubo [24]. L'injection directe d'adénovirus recombinant, déleté des régions $\varepsilon l$ et $\varepsilon 3$, dans lequel on a inséré I'ADNc de la BMP-2 n'entraîne pas de formation osseuse en l'absence d'immunosuppression. En revanche, une immunosuppression transitoire est suffisante pour observer une formation osseuse au site d'injection. Musgrave [25] observe une simple diminution de la formation osseuse en l'absence d'immunosuppression. 


\section{Matrices plasmidiques}

L'utilisation de supports chargés d'un transgène a été proposée. Dans ce scénario, l'ADN d'un plasmide porteur du transgène est chargé sur un matériau support et le dispositif est implanté au niveau de la lésion. Les cellules du patient vont alors ingérer l'ADN du plasmide et exprimer le transgène. Cette stratégie est plus sûre que l'utilisation de vecteurs viraux. Des auteurs ont pu montrer, en utilisant des gènes rapporteurs, qu'effectivement les cellules du tissu de granulation intégraient le plasmide [26]. Les résultats obtenus avec cette stratégie lors du comblement de pertes de substance osseuse chez le rat ou le chien semblent prometteurs [27].

\section{Applications thérapeutiques}

Les applications thérapeutiques des BMP qui sont envisagées concernent essentiellement le comblement de pertes de substance osseuse, le traitement de pseudarthroses et d'arthrodèses rachidiennes. Dans le traitement des pseudarthroses, l'adjonction de BMP humaine à des autogreffes ou à des allogreffes a permis d'obtenir un taux de consolidation voisin de $100 \%$ chez des patients ayant, pour certains, des pertes de substance osseuse atteignant $13 \mathrm{~cm}$ de long [28-30] (pour revue, voir [12]). Le délai moyen de consolidation a varié de 4 à 6 mois, et une seule injection a été nécessaire dans la majorité des cas. Dans le cas des arthrodèses du rachis, on cherche à faire fusionner plusieurs vertèbres entre elles, en interposant un greffon autologue entre les corps vertébraux (arthrodèse inter-somatique), ou entre les apophyses transverses (arthrodèse inter-apophysaire). Le taux d'échec des arthrodèses par autogreffe reste actuellement élevé. L'utilisation des BMP pourrait donc être d'un grand intérêt dans ces indications [31].

En 1995, les industriels du domaine espéraient terminer les essais cliniques en 3 à 4 ans [32]. Pourtant ce n'est que récemment qu'ils ont obtenu l'accord des instances sanitaires. Sofamor-Danek a reçu en 2002 l'accord de la FDA pour utiliser la BMP-2 dans le cadre de fusion intersomatique lombaire. En Europe, Stryker Biotech a obtenu en 2001 une AMM pour la BMP7/0P-1 dans le cadre limité du traitement des fractures non consolidées du tibia, secondaires à un traumatisme chez l'adulte dans les cas où un traitement antérieur par autogreffe a échoué ou quand l'utilisation d'une autogreffe est impossible. Le rapport européen public d'évaluation conclut à des résultats quasi équivalents entre l'autogreffe et la BMP-7. Ces résultats encourageants doivent être poursuivis par des études cliniques multicentriques.

\section{Matériaux ostéogènes}

La conception de substituts ou d'équivalents bio-artificiels d'organes ou de tissus associant un support matriciel à une ou plusieurs composantes cellulaires a été initialemement développée dans les années 1980 et illustrée par la mise au point de substituts cutanés [33] - notamment en France - et de pancréas [34] et de foie bio-artificiels [35]. Ce n'est qu'ultérieurement que cette technique a été transposée à l'os. Dans ce cas, on transplante, au niveau de la lésion, des cellules ostéocompétentes. Ces cellules ne sont pas injectées directement au niveau de la lésion mais préalablement mises en place dans un matériau support. Les cellules utilisées sont des cellules souches mésenchymateuses. Nous avons relaté dans un article récent les avancées dans ce domaine [36]. Nous nous contenterons ici d'illustrer l'intérêt de cette technique. L'intérêt thérapeutique de cette approche a été démontré chez le petit animal par une greffe dans un site ectopique [37] puis au niveau d'une lésion importante du fémur [38, 39], les cellules souches mésenchymateuses étant préalablement ensemencées dans un biomatériau poreux. Toutefois, la pertinence clinique de ces résultats reste discutable au vu de la petite taille des défauts osseux créés. Des études précliniques chez le gros animal et concernant des os porteurs ont été alors effectuées. La difficulté réside essentiellement dans la mise au point d'un matériau support. Ce matériau doit répondre à un cahier des

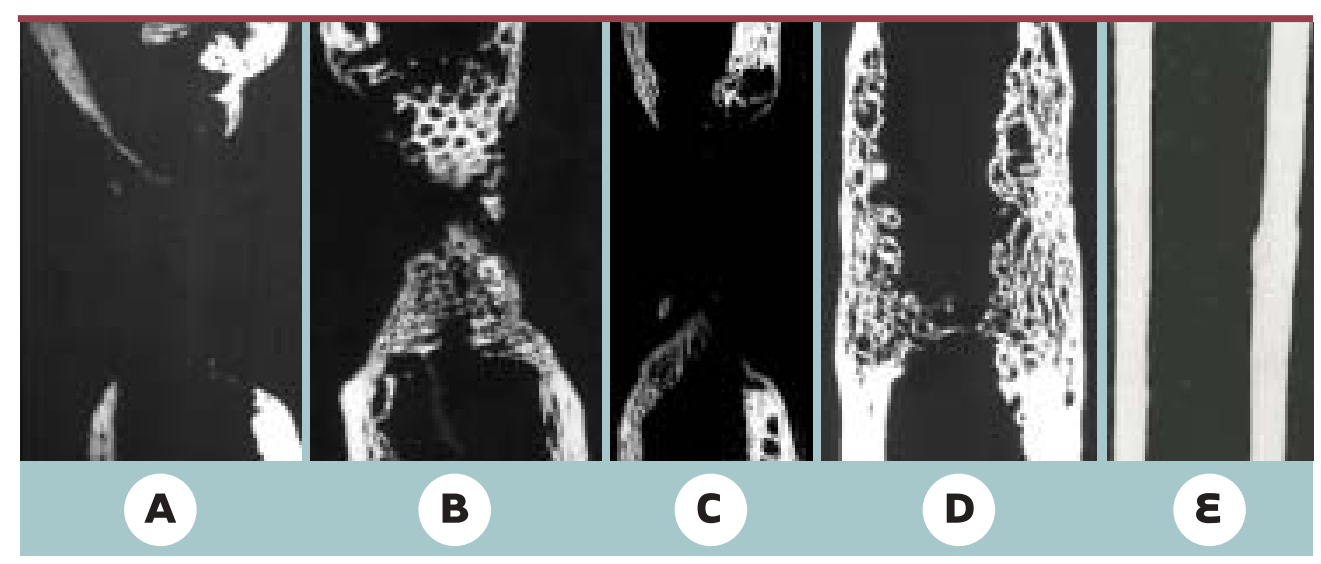

Figure 3. Microradiographies des métatarsiens de brebis 16 semaines après l'implantation de corail. Des défauts non comblés $(A)$ ou comblés avec du corail seul $(B)$, du corail et de la moelle fraîche (C) ne sont pas réparés après 16 semaines. Un défaut osseux comblé avec du corail associé à des cellules souches mésenchymateuses (D) a consolidé après 16 semaines. Un remodelage est nécessaire afin d'obtenir la structure normale de l'os controlatéral $(\varepsilon)$. 
charges exigeant : les ostéoblastes doivent pouvoir proliférer et se différencier sur la matrice ; une néovascularisation doit se développer dans les pores du biomatériau ; ces pores doivent être interconnectés afin de favoriser une angiogenèse rapide et complète de l'implant. On préférera utiliser un matériau résorbable, dont la résorption est concomitante de l'ostéogenèse, de manière à profiter, au départ, des propriétés mécaniques du matériau et à obtenir in fine son remplacement complet par du tissu osseux. Des céramiques à base d'hydroxyapatite [40] ou d'hydroxyapatite/ $\beta$-TCP [41] ont été utilisées. Elles s'avèrent peu résorbables et fragiles. II s'agit plus d'une repousse osseuse à la surface de l'implant que d'une véritable régénération osseuse. Nous avons obtenu des résultats encourageants mais sub-optimaux en utilisant l'exosquelette de corail du genre Porites comme support. Nous l'avons utilisé seul ou après adjonction de moelle osseuse fraîche, ou de cellules souches mésenchymateuses, pour combler des défauts osseux de $25 \mathrm{~mm}$ chez le mouton au niveau d'un os chargé mécaniquement, le métatarsien [42]. II n'y a aucun rétablissement de la continuité osseuse lorsque les défauts osseux sont laissés vacants ou sont comblés par du corail seul ou du corail et de la moelle osseuse fraîche. $\varepsilon$ n revanche, on obtient un rétablissement de la continuité osseuse par au moins une corticale dans 6 cas sur 7 lorsque le corail est ensemencé avec des cellules souches mésenchymateuses (Figure 3 ). Ces résultats très prometteurs doivent cependant être encore améliorés : nous n'avons observé de consolidation clinique satisfaisante que dans 3 cas sur 7 , et il faudra aussi démontrer que l'os néoformé est authentique dans sa composition chimique et qu'il présente des propriétés mécaniques suffisantes.

\section{Conclusions}

Les avancées récentes de la biologie permettent d'entrevoir de nouvelles stratégies thérapeutiques en orthopédie. Ces techniques présenteront vraisemblablement un pouvoir ostéogène très supérieur à celui des biomatériaux classiques. Des questions de sécurité restent pourtant posées et si, bien souvent, on a effectué la démonstration de leur efficacité chez l'animal sain, il n'existe généralement pas d'études cliniques rigoureuses démontrant leur intérêt médical véritable en situation pathologique. On ne peut s'empêcher, par exemple, de se poser la question de l'intérêt de facteurs de croissance ou de cellules souches dans des territoires peu vascularisés. Les études doivent être poursuivies afin de permettre au clinicien de mieux cerner les indications thérapeutiques respectives de ces nouvelles techniques. Malgré ces restrictions, il semble que l'homme soit en train d'accomplir un vieux rêve, celui de reconquérir la capacité de régénérer. On a d'ailleurs

déjà créé un nouveau domaine : celui de la médecine régénératrice. Les premiers balbutiements concernent la régénération de structures simples comme l'os. Gageons que même le plus raisonnable des biologistes sera enthousiaste devant les capacités des urodèles à régénérer des structures complexes à partir d'un blastème. Espérons qu'il y aura un biologiste moins raisonnable pour explorer la possibilité de fabriquer un équivalent du blastème chez les mammifères. $\diamond$

\section{REMERCIEMENTS}

L'auteur tient à remercier Geneviève Guillemin pour sa relecture attentive et Guy Daculsi pour ses éclaircissements sur les phosphates de calcium. Le travail de thérapie cellulaire a bénéficié $d u$ support de la Fondation de l'Avenir pour la Recherche Médicale, du Cnrs et de l'Inserm.

\section{SUMMARY}

\section{Tissue engineering of bone}

Whereas most bone defects will heal spontaneously, 5 to $10 \%$ will require further treatment for compromised healing. In addition, there are specific clinical settings in which large pieces of bone must be resected. In these challenging situations, autologous bone graft is the preferred treatment to obtain bone union. However, autologous bone cannot always be harvested in sufficient amount and has several limitations. Alternatively, surgeons can use osteoconductive bone substitutes but their success is limited as they only provide a scaffold, which needs to be invaded by bone forming cells to achieve bone repair. Recent advances in cell and molecular biology have opened new avenues for improving the osteogenic potential of these materials including their supplementation with either mesenchymal stem cells or growth factors. Whereas preliminary clinical studies have shown that these strategies could be interesting alternatives, further prospective randomized trials are needed to objectively compare them to established treatment. $\diamond$

\section{RÉFÉRENCES}

1. Brockes JP. Amphibian limb regeneration: rebuilding a complex structure. Science $1997 ; 276: 81-7$.

2. Borgens RB. Mice regrow the tips of their foretoes. Science $1982 ; 217$ : 747-50.

3. Einhorn TA. Enhancement of fracture-healing. J Bone Joint Surg Am 1995; 77 : 940-56.

4. Damien C, Parsons R. Bone graft and bone graft substitutes: a review of current technology and applications. J Appl Biomater 1991 ; 2 : 187-208.

5. Goldberg VM, Stevenson S. Natural history of autografts and allografts. Clin Orthop 1987 ; 225 : 7-16.

6. Shors $\varepsilon C$. Coralline bone graft substitutes. Orthop Clin North Am 1999 ; 30 : 599-613. 
7. Duguy N, Petite H, Arnaud

$\varepsilon$. Biomaterials and osseous regeneration. Ann Chir Plast Esthet $2000 ; 45$ : 364-76.

8. Daculsi G, Passuti N, Martin $S$, et al. Macroporous calcium phosphate ceramic for long bone surgery in humans and dogs. Clinical and histological study. J Biomed Mater Res 1990 ; 24 : 379-96.

9. Daculsi G, Bouler JM, LeGeros RZ. Adaptive crystal formation in normal and pathological calcifications in synthetic calcium phosphate and related biomaterials. Int Rev Cytol 1997 ; 172 : 129-91.

10. Frayssinet $\mathrm{P}$, Fages J, Bonel $G$, Rouquet N.

Biotechnology, material sciences and bone repair. Eur J Orthop Surg Traumatol $1998 ; 8: 17-25$.

11. Reddi AH. Role of morphogenetic proteins in skeletal tissue engineering and regeneration. Nat Biotechnol 1998; 16 : 247-52.

12. Li RH, Wozney JM. Delivering on the promise of bone morphogenetic proteins. Trends Biotechnol 2001; 19 : 255-65.

13. Uludag $H$, D’Augusta D, Palmer R, Timony G, Wozney J. Characterization of rhbmp-2 pharmacokinetics implanted with biomaterial carriers in the rat ectopic model. J Biomed Mater Res $1999 ; 46: 193-202$.

14. Groeneveld $\varepsilon H$, Burger $\varepsilon H$. Bone morphogenetic proteins in human bone regeneration. Eur J Endocrinol 2000 ; 142 : 9-21.

15. Arnaud $\varepsilon$, De Pollak C, Meunier A, et al. Osteogenesis with coral is increased by BMP and BMC in a rat cranioplasty. Biomaterials $1999 ; 20$ : 1909-18.

16. Toriumi DM, Kotler HS, Luxenberg DP, Holtrop ME, Wang EA. Mandibular reconstruction with a recombinant bone-inducing factor. Functional, histologic, and biomechanical evaluation.
Arch Otolaryngol Head Neck Surg 1991; 117 : 1101-12.

17. Gerhart TN, Kirker-Head CA, Kriz MJ, et al. Healing segmental femoral defects in sheep using recombinant human bone morphogenetic protein. Clin Orthop 1993; $293: 317-26$.

18. Winn SR, Hu Y, Sfeir C, Hollinger J0. Gene therapy approaches for modulating bone regeneration. Adv Drug Deliv Rev 2000 ; 42 : 121-38.

19. Musgrave DS, Bosch $P$, Lee $J y$, et al. Ex vivo gene therapy to produce bone using different cell types. Clin Orthop 2000 ; 378 : 290-305.

20. Lieberman JR, Daluiski A, Stevenson $\mathrm{S}$, et al. The effect of regional gene therapy with bone morphogenetic protein-2producing bone-marrow cells on the repair of segmental femoral defects in rats. J Bone Joint Surg Am 1999; 81 : 905-17.

21. Krebsbach PH, Gu K, Franceschi RT, Rutherford RB. Gene therapy-directed osteogenesis: BMP-7transduced human fibroblasts form bone in vivo. Hum Gene Ther 2000 ; $11: 1201-10$.

22. Alden TD, Pittman DD, Beres $\varepsilon$, et al. Percutaneous spinal fusion using bone morphogenetic protein-2 gene therapy. J Neurosurg 1999 ; 90 : 109-14.

23. Helm GA, Alden TD, Beres $\varepsilon$,, et al. Use of bone morphogenetic protein-9 gene therapy to induce spinal arthrodesis in the rodent. J Neurosurg 2000; 92 : 191-6.

24. Okubo Y, Bessho K, Fujimura K, lizuka T, Miyatake SI. Osteoinduction by bone morphogenetic protein-2 via adenoviral vector under transient immunosuppression. Biochem Biophys Res Commun $2000 ; 267$ : 382-7.

25. Musgrave DS, Bosch $P$, Ghivizzani S, et al. Adenovirus-mediated direct gene therapy with bone morphogenetic protein- 2 produces bone. Bone 1999; $24: 541-7$.
26. Fang J, Zhu YY, Smiley $\varepsilon$, et al. Stimulation of new bone formation by direct transfer of osteogenic plasmid genes. Proc Natl Acad Sci USA 1996 ; 93 : 5753-8.

27. Goldstein SA, Bonadio J. Potential role for direct gene transfer in the enhancement of fracture healing. Clin Orthop 1998 ; 355 (suppl) : S154-62.

28. Johnson $\varepsilon \varepsilon$, Urist MR, Finerman GA. Distal metaphyseal tibial nonunion. Deformity and bone loss treated by open reduction, internal fixation, and human bone morphogenetic protein (HBMP). Clin Orthop 1990 ; $250: 234-40$

29. Johnson $\varepsilon \varepsilon$, Urist $M R$, Finerman GA. Bone morphogenetic protein augmentation grafting of resistant femoral nonunions. A preliminary report. Clin Orthop 1988; $236: 257-65$.

30. Johnson $\varepsilon \varepsilon$, Urist MR, Finerman GA. Resistant nonunions and partial or complete segmental defects of long bones. Treatment with implants of a composite of human bone morphogenetic protein (BMP) and autolyzed, antigenextracted, allogeneic (AAA) bone. Clin Orthop 1992 ; $280: 229-37$.

31. Helm GA, Alden TD, Sheehan JP, Kallmes D. Bone morphogenetic proteins and bone morphogenetic protein gene therapy in neurological surgery: a review. Neurosurgery $2000 ; 46$ : 1213-22.

32. Alper J. Boning up: newly isolated proteins heal bad breaks. Science $1994 ; 263$ : 324-5.

33. Dubertret L, Coulomb B. Le modèle de peau équivalente. In : Baquey C, Dupuy B, eds. Organes artificiels hybrides. Paris : Éditions Inserm, 1989 : 91-102.

34. Reach G. Pancréas bioartificiel: définition des objectifs et réalisations techniques. In : Baquey C, Dupuy B, eds. Organes artificiels hybrides. Paris :
Éditions Inserm, 1989 :

191-207.

35. Guillouzo A, Gripon P, Guyomard C, et al. Culture d'hépatocytes isolés: utilisation comme modèle alternatif ou substitut fonctionnel. In : Baquey C, Dupuy B, eds. Organes artificiels hybrides. Paris : Inserm, 1989 : 247-55.

36. Petite H. Thérapie cellulaire des grandes pertes de substance osseuse. Med Sci $2001 ; 17: 128-30$.

37. Goshima J, Goldberg VM, Caplan AI. Osteogenic potential of cultureexpanded rat marrow cells as assayed in vivo with porous calcium phosphate ceramic. Biomaterials 1991; $12: 253-8$

38. Kadiyala S, Jaiswal N, Bruder S. Culture-expanded bone marrow-derived mesenchymal stem cells can regenerate a critical-sized segmental bone defect. Tissue Engin 1997 ; 3 : 173-85.

39. Bruder SP, Kurth AA, Shea M, et al. Bone regeneration by implantation of purified, culture-expanded human mesenchymal stem cells. J Orthop Res $1998 ; 16$ : 155-62.

40. Kon $\varepsilon$, Muraglia A, Corsi A, et al. Autologous bone marrow stromal cells loaded onto porous hydroxyapatite ceramic accelerate bone repair in critical-size defects of sheep long bones. J Biomed Mater Res 2000 ; 49 : 328-37.

41. Bruder SP, Kraus KH, Goldberg VM, Kadiyala S. The effect of implants loaded with autologous mesenchymal stem cells on the healing of canine segmental bone defects. J Bone Joint Surg Am 1998 ; $80:$ 985-96.

42. Petite H, Viateau V, Bensaid $W$, et al. Tissue-engineered bone regeneration. Nat Biotechnol $2000 ; 18$ : 959-63.

\section{TIRÉS À PART}

H. Petite 